\title{
Very High Power Very Short Duration Ablation for Atrial Fibrillation: with great power comes great responsibility
}

\author{
Dhiraj Gupta ${ }^{1}$ and Peter Calvert ${ }^{1}$ \\ ${ }^{1}$ Liverpool Heart and Chest Hospital
}

February 28, 2022

\begin{abstract}
vHPvSD ablation represents a quantum leap from the RF settings that have traditionally been used in electrophysiology. If used to its full potential, it may improve procedural efficiency by reducing ablation and procedure times. However, with great power comes great responsibility; we need to ensure that we use it judiciously and safely.
\end{abstract}

\section{EDITORIAL}

Very High Power Very Short Duration Ablation for Atrial Fibrillation: with great power comes great responsibility

Short Title: Very high power short duration ablation

Key words: HPSD ablation, 90 watts ablation, QDot, QMode

Dhiraj Gupta ${ }^{1}$ MD

Peter Calvert ${ }^{1} \mathrm{MBChB}$

${ }^{1}$ Liverpool Centre for Cardiovascular Science, University of Liverpool and Liverpool Heart \& Chest Hospital Liverpool, UK

Correspondence:

Professor Dhiraj Gupta

Professor of Cardiac Electrophysiology / Consultant Cardiologist \& Electrophysiologist

Department of Cardiology,

Liverpool Heart and Chest Hospital,

Liverpool, L14 3PE, U.K.

Tel: $+44(0) 1516001616$

Email: dhiraj.gupta@lhch.nhs.uk

Word count: 1562

Funding: None

Conflicts of Interest: DG has received research funding from Biosense Webster, Boston Scientific and Medtronic. 
For all the advances in electrophysiology, the need to create a focal ablation lesion using radiofrequency (RF) energy has remained remarkably constant. However, the recipe for the perfect RF lesion continues to elude us even after almost 40 years of research and clinical experience. As we continue our search for the holy grail of transmurality and durability with assured safety, there has been renewed interest in temperature controlled (TC) ablation. Accurate tip temperature feedback from catheters such as the QDot Micro ${ }^{\mathrm{TM}}$ catheter (Biosense Webster Inc, Irwindale, CA) have allowed for the first time the use of very high power, very short duration (vHPvSD) ablation that utilises $90 \mathrm{~W}$ RF power for just 4 seconds of ablation per lesion, delivered in the TC mode. Ex-vivo experiments have demonstrated that vHPvSD ablation produces larger, shallower, and more homogeneous lesions as compared to standard power-controlled ablation (sRF); characteristics that should be ideal for the thin-walled left atrial tissue. vHPvSD also produces more transmural and contiguous linear lesions with fewer steam pops compared to sRF ablation, which would be particularly useful for ventricular tissue. If these extremely encouraging results could be replicated in the clinical arena, our search for perfect RF lesion creation may be finally over. Are these high hopes being realised?

The clinical feasibility of the vHPvSD approach was first demonstrated in the QDOT-FAST trial, in which 52 patients underwent successful pulmonary vein isolation (PVI) with impressively short mean procedural and ablation times of $105 \mathrm{~min}$ and $8 \mathrm{~min}$ respectively. However, new asymptomatic cerebral lesions (ACLs) were identified in $6(14 \%)$ patients who underwent MRI scanning, with 4 of these occurring despite uninterrupted anticoagulation. While no patients suffered clinically significant neurological effects, and all but one of these ACLs resolved on repeat MRI performed at 1 month, it did throw a spotlight on an issue that needed redressal before this technology could supplant the other well-established RF ablation techniques.

In this edition of the Journal of Cardiovascular Electrophysiology, Mueller and colleagues present a singlecentre experience of safety outcomes in 34 patients undergoing vHPvSD AF ablation. The principal findings of this study are: 1) modest acute efficacy, as seen by disappointing first pass isolation (FPI) rates of $18 \%$ of patients and $54 \%$ of PV pairs; 2) a concerning safety signal in the form of coagulum on the catheter tip in $10(18 \%)$ patients and ACLs on post-procedure MRI in $26 \%$ patients. The same group had described a similarly high rate of catheter tip coagulum (11\%) and ACLs (24\%) in a previous cohort of patients after which several software amendments to the RF generator (nGEN) were made. However, it appears that the underlying issue persists. Whilst Mueller and colleagues deserve credit for sharing their findings, these will cause consternation amongst the electrophysiology community. After all, with vHPvSD we were hoping for an improvement in both procedural efficacy and safety, but the data from Mueller et al. suggest the polar opposite. This editorial piece will attempt to put these new findings in context with other published work in this area, and also with our own observations gleaned from using vHPvSD for the past 18 months.

\section{Efficacy of vHPvSD Ablation}

FPI is an excellent acute indicator of the quality of RF PVI, and it serves as a reliable surrogate for long term success rates. As such it is notable that the reported FPI rates with vHPvSD have been modest, ranging from $18-61 \%$ (Table 1). Rates of early PV reconnection during the waiting period are also disappointingly high. Both of these result in need for additional RF applications for gap elimination, thereby offsetting one potential benefit of using vHPvSD, namely reduction in procedure and RF times. This is paradoxical - if the bench data demonstrate a better lesion profile, why is this not mirrored in terms of acute clinical efficacy?

A few hypotheses bear consideration. Firstly, bench studies are performed under controlled conditions where stable contact for the full 4 second duration of the RF application is virtually guaranteed. This is far removed from the clinical setting, where a combination of respiratory and cardiac motion means that contact is likely to be intermittent. In fact, it can be argued that catheter stability in vHPvSD is even more critical than with sRF, because even brief loss of contact is substantial, in relative terms. For example, 2 sec loss of contact in a standard $20 \mathrm{sec}$ ablation represents just $10 \%$ of the duration, whilst for vHPvSD it presents $50 \%$. To that end, it is possible that the use of full general anaesthesia, with high frequency, low tidal volume ventilation, may improve results with vHPvSD by stabilising catheter contact. Secondly, lesion contiguity is one of the central tenets of the highly successful CLOSE protocol, which in turn relies on accurate placement of automated lesion tags (Visitags). At the moment, vHPvSD is not compatible with the CARTO Visitag software, which 
leads to high variability in placement of the auto-tags depending upon the phase of respiration. This can make accurate tracking of inter-tag distance extremely challenging. Upcoming software enhancements should address this issue. Thirdly, the current approach to vHPvSD utilises a uniform setting of $90 \mathrm{~W} / 4 \mathrm{sec}$ ablation throughout all areas of the ablation circle. It makes no allowance for the well-recognised differences in tissue thickness between the anterior and posterior left atrial regions, unlike the CLOSE protocol that utilises different ablation-index targets for these regions. It may be relevant that the median depth of vHPvSD lesions seen by Takigawa et al. was $2.7 \mathrm{~mm}$, which may not be adequate to produce transmurality across the thick left atrial appendage ridge in all patients. Some operators, including ourselves, have tried to get around this limitation by clustering lesions closer together on the anterior segments. It may be no coincidence that the one group that reported high efficacy with vHPvSD had targeted an inter-tag distance of 3-4mm on the anterior wall. Perhaps that is what may be needed for vHPvSD in place of the standard $6 \mathrm{~mm}$ spacing that was validated on entirely different conventional RF settings.

In summary, whilst more data are needed, it looks likely that the efficacy of vHPvSD ablation may be improved by use of general anaesthesia, and by shortening the inter-tag distance, especially on the anterior segments (Figure 1).

\section{Safety of vHPvSD Ablation}

It is important to note that all published case series utilising vHPvSD are extremely small, with patients ranging from 28 to 90 . Given the relative infrequency of serious complications such as clinical stroke and atriooesophageal complications with AF ablation, these studies are grossly underpowered to provide conclusive safety data. As such, we need to look for surrogate markers of complications, such as oesophageal lesions on endoscopy and asymptomatic cerebral lesions (ACLs) on cranial MRIs.

The incidence of post-procedure oesophageal injury is reassuringly low in the 3 studies that have evaluated this systematically with endoscopy (Table 2). In QDOT-FAST, a haemorrhaging ulcer was seen in just 1 of the 52 patients, and healed with medical therapy. The other two studies, comprising 134 patients, showed no evidence of oesophageal injury in any patient. This reassuring observation is in keeping with the findings of the bench studies; vHPvSD lesions tend to be wider but shallower thereby reducing the potential for extracardiac damage.

However, more worrying are the reports of coagulum formation on the catheter tip and high rates of ACLs which likely represent associated thromboembolic events from this charring (Table 2). Rates of postprocedure ACL in vHPvSD studies have varied from $11.8 \%$ to $26 \%$, which are higher than seen with sRF. These have occurred in spite of appropriate intra-procedural anticoagulation, and even after recent software modifications to the nGEN RF generator. Whilst these ACLs were not associated with clinical stroke events, and most (but not all) resolved on follow-up MRI a few months later, recent prospective data suggest that even silent ACLs can be associated with cognitive decline over a relatively short timeframe. As such, it is clearly preferable to minimise - or prevent entirely - the risk of ACL occurrence. How can we do so?

One possible solution is suggested by Mueller et al. themselves. While they found catheter tip coagulum in almost a third of patients initially (6 out of the first 19 patients), this stopped happening entirely when the baseline circuit impedance was increased - via repositioning of the neutral electrode - from 90 to 110. This interesting observation lends credence to the theory that coagulum formation results from excessively high current flow with lower impedance. Bourier et al. recently demonstrated the critical impact of circuit impedance on ablation, emphasising that it is current delivery, rather than power input, which determines lesion size, and that current delivery can vary widely due to fluctuations in impedance. This effect may be particularly magnified in vHPvSD due to the short duration of current delivery. More research is needed to find the optimal balance of current delivery by modulation of impedance and power, perhaps by development of a 'constant current mode' as suggested by Bourier et al. .

vHPvSD ablation represents a quantum leap from the RF settings that have traditionally been used in electrophysiology. If used to its full potential, it may improve procedural efficiency by reducing ablation and procedure times. However, with great power comes great responsibility; we need to ensure that we use it 
judiciously and safely (Figure 1). To that end, we are grateful to Mueller and colleagues for highlighting these issues for us to work on.

\section{References}

\begin{tabular}{|c|c|c|c|c|c|}
\hline Study & $\begin{array}{l}\text { RF } \\
\text { Generator / } \\
\text { Settings }\end{array}$ & $\begin{array}{l}\text { Inter-tag } \\
\text { distance }\end{array}$ & $\begin{array}{l}\text { First Pass } \\
\text { Isolation } \\
\text { Rate }\end{array}$ & $\begin{array}{l}\text { Total RF } \\
\text { time }(\mathrm{s})\end{array}$ & $\begin{array}{l}\text { Need to use } \\
\text { standard RF } \\
\text { settings, N } \\
(\%)\end{array}$ \\
\hline $\begin{array}{l}\text { QDOT-FAST } \\
(2019) \mathrm{N}=52\end{array}$ & $\begin{array}{l}\text { nMARQ 90W / } \\
4 \text { sec }\end{array}$ & Not reported & Not reported* & 486 & $11(22 \%)$ \\
\hline $\begin{array}{l}\text { Halbfass et } \\
\text { al.(2021) } \mathrm{N}=90\end{array}$ & $\begin{array}{l}\text { nGEN or } \\
\text { nMARQ 90W } \\
4 \text { sec anteriorly } \\
90 \mathrm{~W} 3 \mathrm{sec} \\
\text { posteriorly }\end{array}$ & $\begin{array}{l}6 \mathrm{~mm} \text { anteriorly } \\
6 \mathrm{~mm} \text { posteriorly }\end{array}$ & $\begin{array}{l}43 \% \text { of patients } \\
65 \% \text { of PV pairs }\end{array}$ & Not reported & $51(57 \%)$ \\
\hline $\begin{array}{l}\text { Tilz et al. (2021) } \\
\mathrm{N}=28\end{array}$ & $\begin{array}{l}\text { Not reported } \\
90 \mathrm{~W}, 4 \mathrm{sec}\end{array}$ & $\begin{array}{l}3-4 \mathrm{~mm} \\
\text { anteriorly } 5-6 \\
\mathrm{~mm} \text { posteriorly }\end{array}$ & $\begin{array}{l}61 \% \text { of patients } \\
80 \% \text { of PV pairs }\end{array}$ & 338 & $0(0)$ \\
\hline $\begin{array}{l}\text { Mueller et al. } \\
\text { (2022) N=34 }\end{array}$ & $\begin{array}{l}\text { nGEN (v1c } \\
\text { software) 90W } \\
\text { 4sec anteriorly } \\
90 \mathrm{~W} 3 \mathrm{sec} \\
\text { posteriorly }\end{array}$ & $\begin{array}{l}6 \mathrm{~mm} \text { anteriorly } \\
6 \mathrm{~mm} \text { posteriorly }\end{array}$ & $\begin{array}{l}18 \% \text { of patients } \\
54 \% \text { of PV pairs }\end{array}$ & $774+/-594$ & $29(85 \%)$ \\
\hline
\end{tabular}

Table 1: Studies reporting on the efficacy of very high power very short duration (vHPvSD) ablation for atrial fibrillation. PV - Pulmonary Vein; RF - Radiofrequency.

*First pass isolation figures not explicitly stated; 14(27\%) patients had dormant conduction revealed in 20 sites with Adenosine / Isoproterenol challenge after 20 minutes

\begin{tabular}{|c|c|c|c|c|}
\hline Study & $\begin{array}{l}\text { RF Generator } \\
\text { / Settings }\end{array}$ & $\begin{array}{l}\text { Oesophageal } \\
\text { Injury }\end{array}$ & $\begin{array}{l}\text { Catheter } \\
\text { Coagulum }\end{array}$ & $\begin{array}{l}\text { Cerebral } \\
\text { Lesions }\end{array}$ \\
\hline $\begin{array}{l}\text { QDOT-FAST } \\
(2019) \mathrm{N}=52\end{array}$ & nMARQ 90W / 4sec & $\begin{array}{l}1 \text { ulcer } \\
\text { haemorrhage, } \\
\text { healed with } \\
\text { medication }\end{array}$ & Not reported & $\begin{array}{l}\text { No clinical stroke } \\
11.8 \%(6 / 51) \text { ACL }\end{array}$ \\
\hline $\begin{array}{l}\text { Halbfass et al. } \\
(2021) \mathrm{N}=90\end{array}$ & $\begin{array}{l}\text { nGEN or nMARQ } \\
\text { 90W 4sec anteriorly } \\
90 \mathrm{~W} 3 \mathrm{sec} \\
\text { posteriorly }\end{array}$ & $\begin{array}{l}\text { None found on } \\
\text { endoscopy }\end{array}$ & $\begin{array}{l}\text { None with nMARQ } \\
11 \% \text { with nGEN }\end{array}$ & $\begin{array}{l}\text { No clinical stroke } \\
24 \%(5 / 21) \text { ACL } \\
\text { (only with nGEN) }\end{array}$ \\
\hline $\begin{array}{l}\text { Tilz et al. }(2021) \\
\mathrm{N}=28\end{array}$ & $\begin{array}{l}\text { Not reported } 90 \mathrm{~W} \\
4 \mathrm{sec}\end{array}$ & $\begin{array}{l}\text { No clinical events } \\
\text { (no endoscopy } \\
\text { performed) }\end{array}$ & None & $\begin{array}{l}\text { No clinical stroke } \\
\text { ACL not looked for }\end{array}$ \\
\hline $\begin{array}{l}\text { Mueller et al. } \\
(2022) \mathrm{N}=34\end{array}$ & $\begin{array}{l}\text { nGEN (v1c } \\
\text { software) 90W 4sec } \\
\text { anteriorly 90W } 3 \mathrm{sec} \\
\text { posteriorly }\end{array}$ & $\begin{array}{l}\text { None found on } \\
\text { endoscopy }\end{array}$ & $\begin{array}{l}18 \% \text { with baseline } \\
\text { impedance } 90, \text { none } \\
\text { with } 110\end{array}$ & $\begin{array}{l}\text { No clinical stroke } \\
26 \%(6 / 23) \text { ACL }\end{array}$ \\
\hline
\end{tabular}

Table 2: Studies reporting on the safety of very high power very short duration (vHPvSD) ablation for 
atrial fibrillation. ACL - Asymptomatic Cerebral Lesion; RF - Radiofrequency.

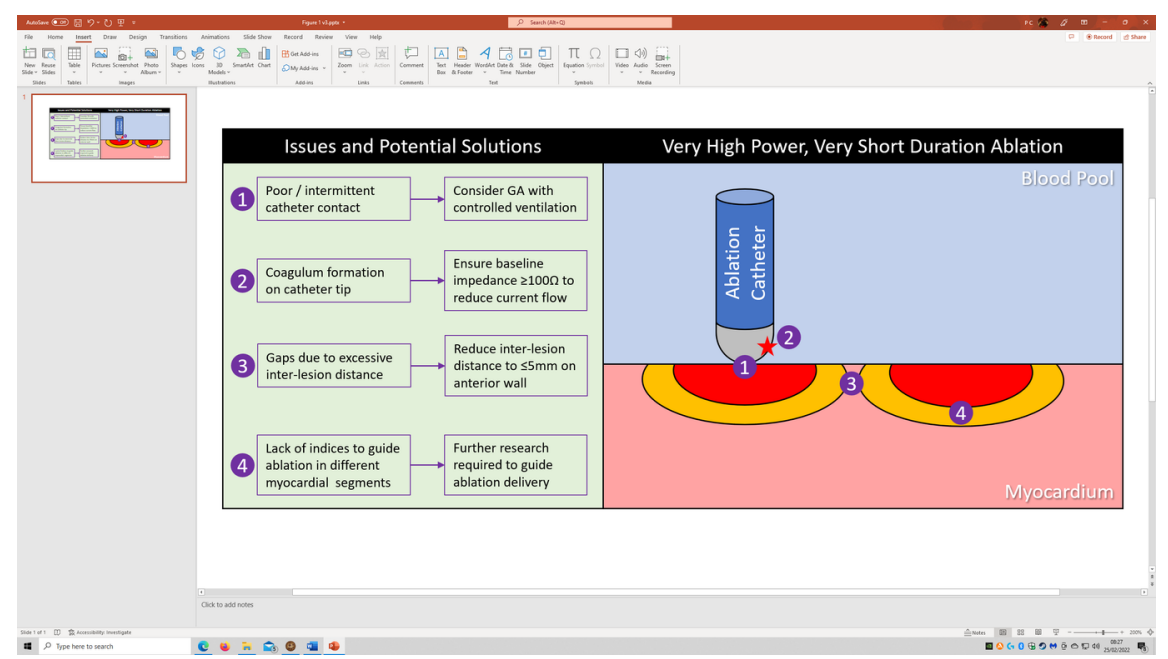

Figure 1: Issues and potential solutions when utilising vHPvSD ablation. GA - general anaesthetic.

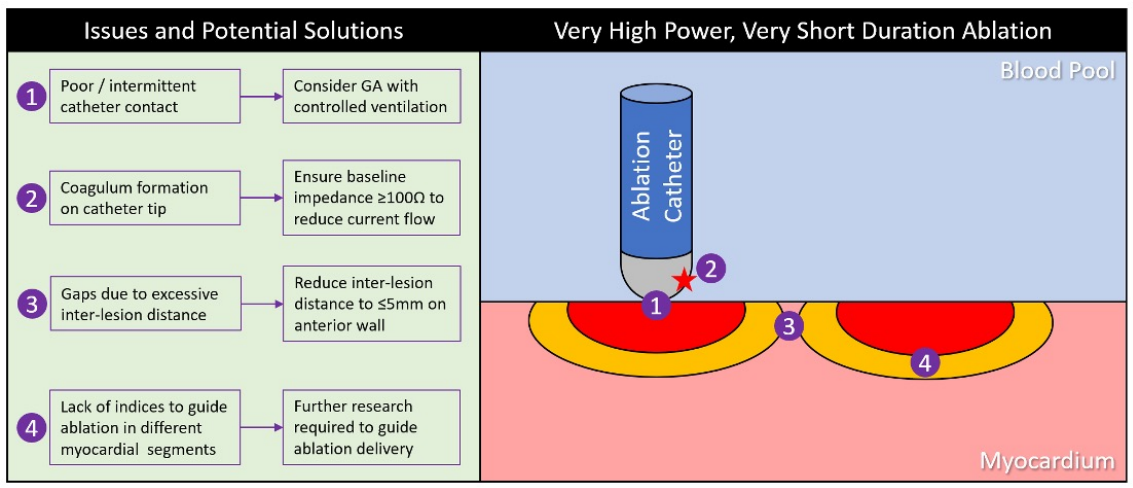

